\title{
Citogenética do javali em criatórios comerciais das regiões Sul e Sudeste do Brasil(1)
}

\author{
Lenícy Lucas de Miranda ${ }^{(2)}$ e Jeffrey Frederico Lui(3) \\ Resumo - O desenvolvimento da criação de javalis no Brasil encontra dificuldades tais como a obtenção \\ de animais puros $(2 \mathrm{n}=36)$, isto é, que não sejam descendentes do cruzamento do javali com o porco \\ doméstico. Os objetivos deste trabalho foram a caracterização citogenética do javali (Sus scrofa scrofa L.) \\ e a determinação da frequiência cariotípica dos diferentes grupos genéticos. Foram estudados um total de \\ 1.308 javalis de três grupos genéticos provenientes de criatórios comerciais das regiões Sul e Sudeste do \\ Brasil, de ambos os sexos e com idade entre 5,5 a 30 meses. Os animais apresentaram número diplóide \\ (2n) de 36 cromossomos $(46,71 \%)$, de $37(39,68 \%)$ e de 38 cromossomos $(13,61 \%)$. Os dados foram \\ submetidos à análise estatística por meio do Teste Exato de Fisher. Na técnica de bandamento C, todos \\ os cromossomos dos animais analisados dos três números diplóides apresentaram banda $\mathrm{C}$ positiva na \\ região do centrômero e apenas o cromossomo Y mostrou-se quase inteiramente heterocromático. Já no \\ bandamento NOR, dois pares de metacêntricos foram corados nas constrições secundárias próximas ao \\ centrômero, correspondendo aos pares 7 e 10 nos animais $2 \mathrm{n}=36,37$ e 38 . A presença de um cromossomo \\ submetacêntrico médio $t(15 ; 17)$, formado por fusão robertsoniana nos animais com $2 n=37$ pode \\ diferenciar javalis híbridos, puros e javaporcos, visto que são fenotipicamente muito parecidos.
}

Termos para indexação: Sus scrofa, cruzamento, cromossomos, seleção, polimorfismo.

\section{Cytogenetics of wild boars from commercial breeders in Southern and Southeastern Brazil}

Abstract - Wild boar breeding in Brazil frequently faces difficulties, such as the acquisition of pure animals $(2 n=36)$, that is, which are not descendants of the wild boar and domestic swine crossings. The purposes of this work were the cytogenetic characterization of the wild boar (Sus scrofa scrofa L.) and the determination of the karyotypic frequency of different genetic groups. One thousand, three hundred and eight wild boars from three genetic groups from the southern and southeastern regions of Brazil were studied. The sample consisted of male and female animals aged 5.5 to 30 months. They showed a diploid number (2n) of $36(46.71 \%), 37(39.68 \%)$ and $38(13.61 \%)$ chromosomes. The data were submitted to statistical analysis through the Fisher's Exact Test. According to the $\mathrm{C}$ banding technique, all the chromosomes of the analyzed animals with three diploid numbers showed positive $\mathrm{C}$ band at the centromere region and only chromosome $\mathrm{Y}$ was almost entirely heterochromatic. However, in NOR banding, two pairs of metacentric chromosomes were stained at the secondary constrictions close to the centromere, corresponding to pairs 7 and 10 in $2 n=36,37$ and 38 animals. The presence of a medium submetacentric chromosome $\mathrm{t}(15 ; 17)$, formed by robertsonian fusion in the animals with $2 \mathrm{n}=37$ can differentiate hybrid pure wild boars and from "javaporcos" as they are phenotypically very similar.

Index terms: Sus scrofa, crossbreeding, chromosomes, selection, polymorphism.

\footnotetext{
(1) Aceito para publicação em 19 de agosto de 2003.

(2) Universidade de São Paulo, Fac. de Medicina de Ribeirão Preto, Dep. de Genética, Av. Bandeirantes, 3900 CEP 14049-900 Ribeirão Preto, SP. E-mail: lenicy@rge.fmrp.usp.br

(3) Universidade Estadual Paulista, Fac. de Ciências Agrárias e Veterinárias, Dep. de Zootecnia, Rodovia Paulo Donato Castellane, km 5, CEP 14870-000 Jaboticabal, SP. E-mail: jeffrey@fcav.unesp.br
}

\section{Introdução}

Os javalis são mamíferos que pertencem à ordem Artiodactyla, da família Suidae, originalmente encontrada na Europa, Ásia e África, representada por cinco gêneros, entre eles o Sus e o Babyrousa (Bosma et al., 1996). De acordo com Nowak (1999), o gênero Sus compreende cinco espécies vivas, entre elas a Sus scrofa L.É uma espécie comum na Eurásia e ocor- 
re no noroeste da África; acredita-se na existência de no mínimo 16 subespécies (Giuffra et al., 2000), como por exemplo, o javali europeu (S. s. scrofa), o porco doméstico (S. s. domestica) e o javali da Malásia e Indonésia (S. s. vittatus) (Chen \& Leibenguth, 1995).

No início do século XX, foram trazidos alguns animais para a Argentina, com o propósito de servir como caça esportiva, que acabaram escapando e se espalharam pelo norte da Argentina, Uruguai e Sul do Brasil. Na década de 90 começaram a ser importados alguns animais puros, para fins de criação comercial, principalmente da França e Canadá. $\mathrm{O}$ interesse pela criação comercial do javali deve-se à sua carne de excelente sabor e ótima qualidade nutricional (Vitic \& Stevanovic, 1993; Wolkers et al., 1993; Andersson-Eklund et al., 1998). Além da carne, são de interesse seus derivados, como presuntos de pernil, paleta, defumados de lombo, patês e embutidos como salames e lingüiças. $\mathrm{O}$ couro pode servir à confecção de casacos e luvas, e as cerdas são utilizadas na Europa na fabricação de pincéis, escovas de cabelo e outros.

A domesticação dos animais selvagens é um importante evento na história da humanidade. Tem sido intrigante identificar os ancestrais selvagens dos animais domésticos e inferir sobre a difusão de uma civilização a outra e sobre a evolução dos animais em processo de domesticação (Huang et al., 1999).

Há perspectiva de expansão da genômica animal e os resultados dessa tecnologia, se combinados com novas técnicas de reprodução, poderão mudar e melhorar a maneira como o criador faz e orienta o melhoramento genético (Rothschild \& Plastow, 1999).

Existem várias diferenças morfológicas entre o javali e o porco doméstico devido aos efeitos da seleção artificial. No javali a cauda é sempre reta, o focinho e as patas são escuros, havendo a presença de crina e pêlos longos no corpo. A altura dos membros torácicos é ligeiramente maior que dos pélvicos, além de ser um animal mais alto e mais curto que o porco doméstico (Nowak, 1999).

O javali apresenta $2 \mathrm{n}=36$ e o porco doméstico $2 n=38$ cromossomos (Bosma, 1976). Atualmente considera-se o cariótipo padrão para o javali europeu (Sus s. scrofa) como sendo 2n = 36 (Darré et al., 1992). Ocorrem também no país animais híbridos com $2 \mathrm{n}=37$ cromossomos, resultantes do cruzamento do javali com o porco doméstico.

O desenvolvimento da criação de javalis em nosso país apresenta dificuldades, tais como a obtenção de animais puros $(2 n=36)$ capturados vivos nos seus ambientes naturais ou oriundos de criatórios comerciais. Isto pode ser sanado pela realização de testes citogenéticos para determinar o número cromossômico dos animais.

Os objetivos deste trabalho foram a caracterização citogenética do javali (Sus scrofa scrofa L.) e a determinação da frequiência cariotípica dos diferentes grupos genéticos.

\section{Material e Métodos}

O trabalho foi realizado no período de dezembro de 1997 a outubro de 2001. Analisaram-se 1.308 javalis (Sus scrofa scrofa L.), chamados de javali europeu, de ambos os sexos, com idades entre 5,5 e 30 meses, provenientes de criatórios comerciais das regiões Sul e Sudeste do Brasil.

A análise citogenética foi feita por meio da cultura de linfócitos do sangue periférico de acordo com a técnica descrita por Moorhead et al. (1960). Cerca de 15 metáfases de cada indivíduo foram analisadas para verificação do seu número diplóide e, conseqüentemente, a classificação em grupos genéticos de acordo com a ploidia encontrada ( $2 \mathrm{n}=36,37$ e 38 cromossomos). Os diferentes números cromossômicos encontrados nas regiões Sul e Sudeste foram submetidos à análise estatística por meio do Teste Exato de Fisher (Zhang et al., 1998). A nomenclatura dos cromossomos, com base na posição do centrômero, foi estabelecida por Levan et al. (1964).

A técnica de formação de bandamento $\mathrm{C}$ foi baseada na de Sumner (1972), ou seja, utilização de $0,02 \mathrm{~N} \mathrm{HCl}$, solução a $0,02 \mathrm{~N}$ de $\mathrm{Ba}(\mathrm{OH})_{2}$ e $2 X$ SSC (solução salina citratada).

A técnica de formação de bandamento NOR foi baseada na de Goodpasture \& Bloom (1975), utilizando-se impregnação pelo $\mathrm{AgNO}_{3}$ na região organizadora do nucléolo.

\section{Resultados e Discussão}

Nos 1.308 javalis analisados citogeneticamente nas regiões Sul (211 indivíduos) e Sudeste (1.097 indivíduos) do Brasil, foram encontradas três diferentes ploidias $(2 \mathrm{n}=36,37$ e 38 cromossomos). O número de indivíduos híbridos $(2 \mathrm{n}=37)$ apesar de ter sido maior na região Sul, não se mostrou significativo por meio do Teste Exato de Fisher, provavelmente em virtude do tamanho da amostra ser menor que a da região Sudeste. As demais comparações, $2 n=36$ e $2 n=38$ nas regiões Sul e Sudeste, mostraram-se significativas (Tabela 1). Esses resultados são, em parte, semelhantes aos de Darré et al. (1992) na França, onde a proporção de javalis com 37 ou 38 cromossomos diferiu muito de acordo com a população, região e direção de acasalamento. 
Segundo a disposição do centrômero, o tamanho dos cromossomos e de acordo com a orientação do padrão cariotípico, previamente estabelecido para o suíno doméstico por Gustavsson (1988), pôde-se classificar os cromossomos de javalis machos puros $(2 n=36)$ da seguinte maneira: o par 1 é um submetacêntrico grande; o part $(15 ; 17)$ e os pares 2 a 4 são submetacêntricos medianos; os pares 5 e 6 são subtelocêntricos medianos. O cromossomo X é um submetacêntrico mediano e o cromossomo Y um metacêntrico pequeno. Os pares 7 a 12 são metacêntricos pequenos; o par 13 é um telocêntrico mediano e os pares 14,16 e 18 são telocêntricos pequenos (Figura 1a).

O cariótipo com $2 \mathrm{n}=38$ cromossomos (Figura $1 \mathrm{~b}$ ) dos animais chamados javaporcos foi montado seguindo o mesmo padrão, sendo o par 1 um submetacêntrico grande, os pares 2 a 4 submetacêntricos medianos; os pares 5 e 6 são subtelocêntricos medianos. $\mathrm{O}$ cromossomo sexual X é um submetacêntrico médio e o cromossomo Y um metacêntrico pequeno. Os pares 7 a 12 são metacêntricos pequenos. $O$ par 13 é um telocêntrico mediano e os pares 14 a 18 são telocêntricos pequenos.

O cariótipo com $2 \mathrm{n}=37$ cromossomos (Figura 1c), considerado como híbrido, foi montado obedecendo ao mesmo padrão previamente estabelecido, sendo o par 1 um submetacêntrico grande, os pares 2 a 4 são submetacêntricos medianos e os pares 5 e 6 , subtelocêntricos medianos. $\mathrm{O}$ cromossomo sexual $\mathrm{X}$ é um submetacêntrico médio e o cromossomo Y, um metacêntrico pequeno. $\mathrm{O}$ cromossomo $\mathrm{t}(15 ; 17)$ é um submetacêntrico médio formado pela fusão robertsoniana de um dos cromossomos do par 15 com um dos cromossomos do par 17, ambos telocêntricos. Os pares 7 a 12 são metacêntricos pequenos. O par 13 é um telocêntrico mediano; os pares 14,16 e 18 e os cromossomos 15 e 17 são telocêntricos pequenos.

De acordo com Darré et al. (1992), a diferença cromossômica entre o porco e o javali europeu provém de uma fusão robertsoniana entre os

Tabela 1. Distribuição, segundo as ploidias (2n), de uma amostra de 211 javalis da região Sul e 1.097 javalis da região Sudeste.

\begin{tabular}{lcccc}
\hline Região & $2 \mathrm{n}=36$ & $2 \mathrm{n}=37$ & $2 \mathrm{n}=38$ & Total \\
\hline Sul & 78 & 89 & 44 & 211 \\
Sudeste & 537 & 428 & 132 & 1097 \\
$\mathrm{p}^{(1)}$ & $0,027^{*}$ & $0,309{ }^{\mathrm{ns}}$ & 0,032 & - \\
\hline${ }^{(1)}$ Teste exato de Fisher. ${ }^{\text {ns }}$ Não-significativo. *Significativo a $5 \%$.
\end{tabular}

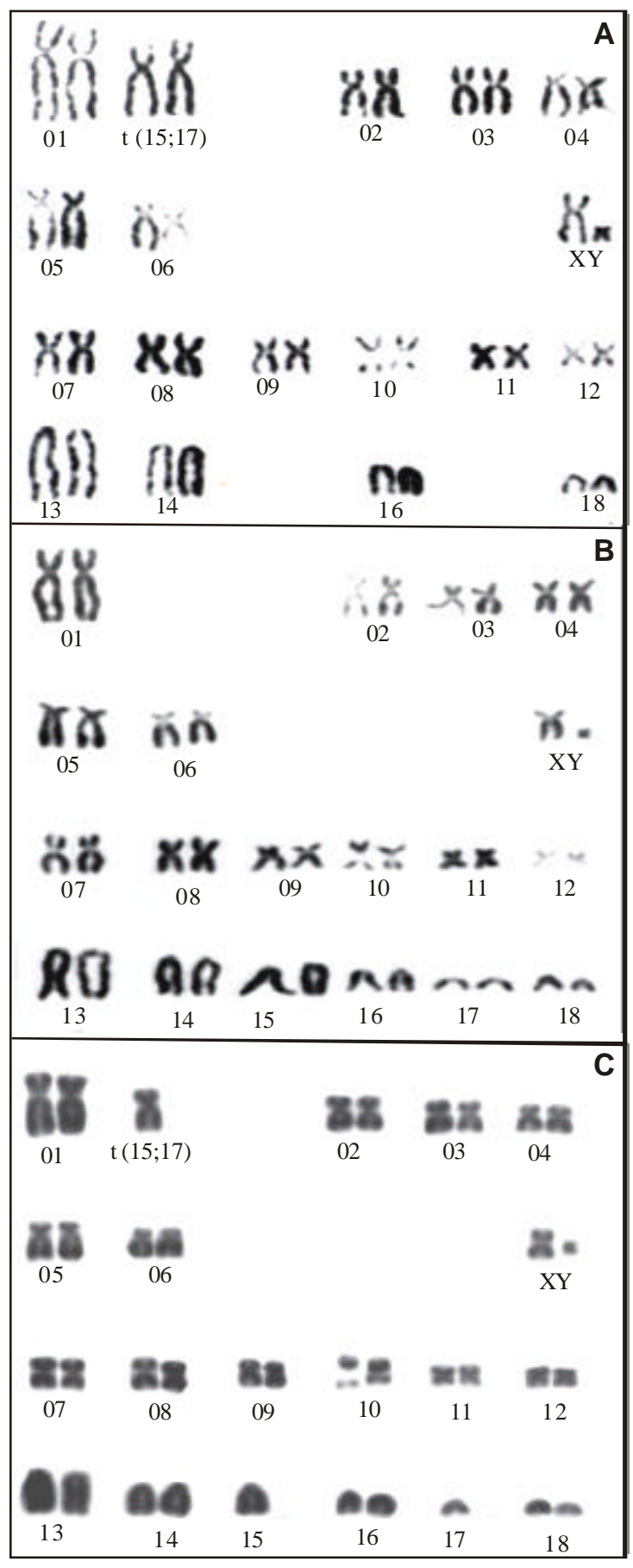

Figura 1. Cariótipo de Sus s. scrofa L. machos, sob coloração convencional, apresentando $2 \mathrm{n}=36$ cromossomos (A); $2 \mathrm{n}=38$ cromossomos (B) e $2 \mathrm{n}=37$ cromossomos $(\mathrm{C})$. 
cromossomos 15 e 17 . O complemento cromossômico do javali $(2 \mathrm{n}=36$ cromossomos $)$ diferiu daquele do javaporco $(2 n=38)$ por exibir um par de submetacêntricos resultantes da fusão robertsoniana dos cromossomos 15 e 17 . O híbrido com 37 cromossomos contém apenas um cromossomo submetacêntrico formado pela fusão dos cromossomos 15 e 17. Porém, a ausência do bandamento $G$ neste trabalho não permitiu identificar as homologias correspondentes. Evidências apresentadas por Thomsen et al. (1996) sugerem que o cromossomo 3 do suíno doméstico é derivado de uma fusão telômero-centrômero dos cromossomos homólogos 12 e 17 do seu ancestral babirussa (Babyrousa babyrussa).

O padrão cariotípico determinado para os três grupos genéticos diferiu da maioria dos padrões encontrados, como por exemplo o de Darré et al. (1992), principalmente quanto à disposição do cromossomo t $(15 ; 17)$.

$\mathrm{O}$ número fundamental (número de braços cromossômicos) manteve-se constante nos cariótipos $2 \mathrm{n}=36,37 \mathrm{e} 38(\mathrm{NF}=64)$. Issoé explicado pelofato de que ocorreram fusões ou fissões nos javalis estudados, enquanto mudanças no número de braços são atribuídas às inversões, o que não foi observado neste trabalho.

Estudos citogenéticos envolvendo os porcos selvagens europeus e asiáticos, que incluem a espécieSusscrofa, comumente conhecidos por javalis, tiveram início na década de 60, sendo encontrados números diplóides (2n), variando entre 36 e 38 cromossomos (McFee et al., 1966; McFee \& Banner, 1969).

Atualmente é considerado, pelos criadores e por alguns autores (Darré et al., 1992), o cariótipo de 2n $=36$ como padrão para o javali europeu (Sus s. scrofa). Os cruzamentos do tipo $36 \times 37,37$ × 38, 36 × 38, 37 × 37 e $38 \times 38$ podem resultar em híbridos com $2 \mathrm{n}=37 \mathrm{e}$ javaporcos com $2 n=38$. Os animais $2 n=36$, resultantes desses cruzamentos, são considerados cariotipicamente como javalis. O polimorfismo cromossômico encontrado corrobora os trabalhos de Bosma et al. (1983, 1991) e Darré et al. (1992).

A presença de um número diplóide de 38 cromossomos em $S$. salvanius pode ser usada na discussão sobre o número cromossômico básico no gênero Sus. Há uma aparente mudança geográfica no número de cromossomos no $S$. scrofa selvagem de leste $(2 n=38)$ a oeste $(2 n=36)$. As diferenças no número cromossômico refletem a presença de um polimorfismo balanceado por translocações robertsonianas nestas espécies (Bosma et al., 1983).
Bosma et al. (1991) realizaram um estudo citogenético comparativo entre Sus verrucosus, Sus celebensis e Sus scrofa vittatus e encontraram um número diplóide de 38 cromossomos, o último com cariótipo idêntico ao do suíno doméstico. Esses autores acreditam que a presença de $2 \mathrm{n}=38$ em Sus verrucosus, e Sus celebensis produz uma evidência nova e forte de que o número cromossômico básico do gênero Sus é 38. Em Panda et al. (1995), o porco selvagem (macho) identificado como javali asiático (Sus scrofa cristatus) exibiu um número diplóide de 38 cromossomos similar ao registrado para o javali japonês (S. s. leucomystax) e javali asiático (S. s. vittatus).

Segundo Tikhonov \& Troshina (1978), cruzamentos entre suínos domésticos (S. s. domestica) com $2 \mathrm{n}=38$ e porcos selvagens ( $S$. s. nigripes e S. s. scrofa) $\operatorname{com} 2 \mathrm{n}=36$ cromossomos têm produzido grande número de híbridos viáveis e férteis durante as quatro primeiras gerações estudadas. O porco híbrido estudado apresentou mudanças no cariótipo não apenas com respeito ao número cromossômico, mas também aos grupos de ligação.

$\mathrm{Na}$ técnica de bandamento $\mathrm{C}$ todos os cromossomos dos animais analisados apresentaram banda $\mathrm{C}$ na região do centrômero. $\mathrm{O}$ cromossomo $\mathrm{Y}$ mostrou-se quase inteiramente heterocromático como é comum na maioria dos mamíferos (Figura 2).

É frequiente encontrar diferentes tamanhos das bandas $\mathrm{C}$ entre os cromossomos homólogos, como pôde ser observado neste trabalho, e talvez isto se deva a variações do conteúdo da heterocromatina constitutiva, que, em virtude de ser formada por sequiência de DNA repetitivo, ficaria sujeita a modificações sem prejuízo da informação genética. Não foram observadas diferenças marcantes entre os três diferentes números diplóides quanto à coloração da heterocromatina constitutiva.

Em Bosma et al. (1991), a morfologia e o tamanho da banda $\mathrm{C}$ do cromossomo 10 foram diferentes em $S$. verrucosus e as outras espécies (S. celebensis e S. s. vittatus). A evolução cromossômica do gênero Sus parece estar acompanhada por trocas nas regiões positivas para a banda $\mathrm{C}$ dos cromossomos, em adição a ocorrência de translocações do tipo fusão cêntrica.

As metáfases submetidas à técnica de formação de bandamento NOR apresentaram marcações em dois pares de cromossomos, pares 7 e 10 nos animais $\operatorname{com} 2 \mathrm{n}=36,38$ e 37 , na região centromérica (Figura 3). Os resultados encontrados diferiram dos de Giannoni et al. (1980), em que a marcação aparece 


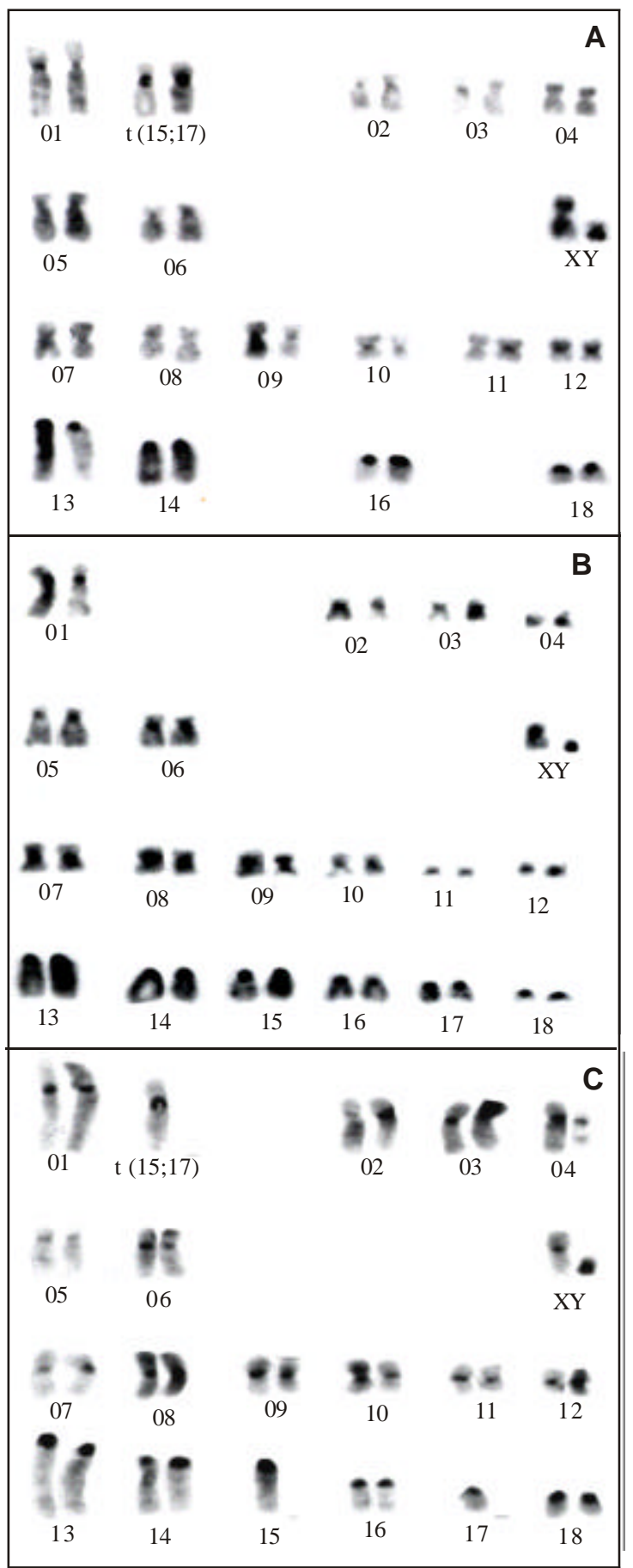

Figura 2. Cariótipo de Sus s. scrofa L. machos, sob bandamento $\mathrm{C}$, apresentando $2 \mathrm{n}=36$ cromossomos (A); $2 n=38$ cromossomos (B) e $2 n=37$ cromossomos (C).

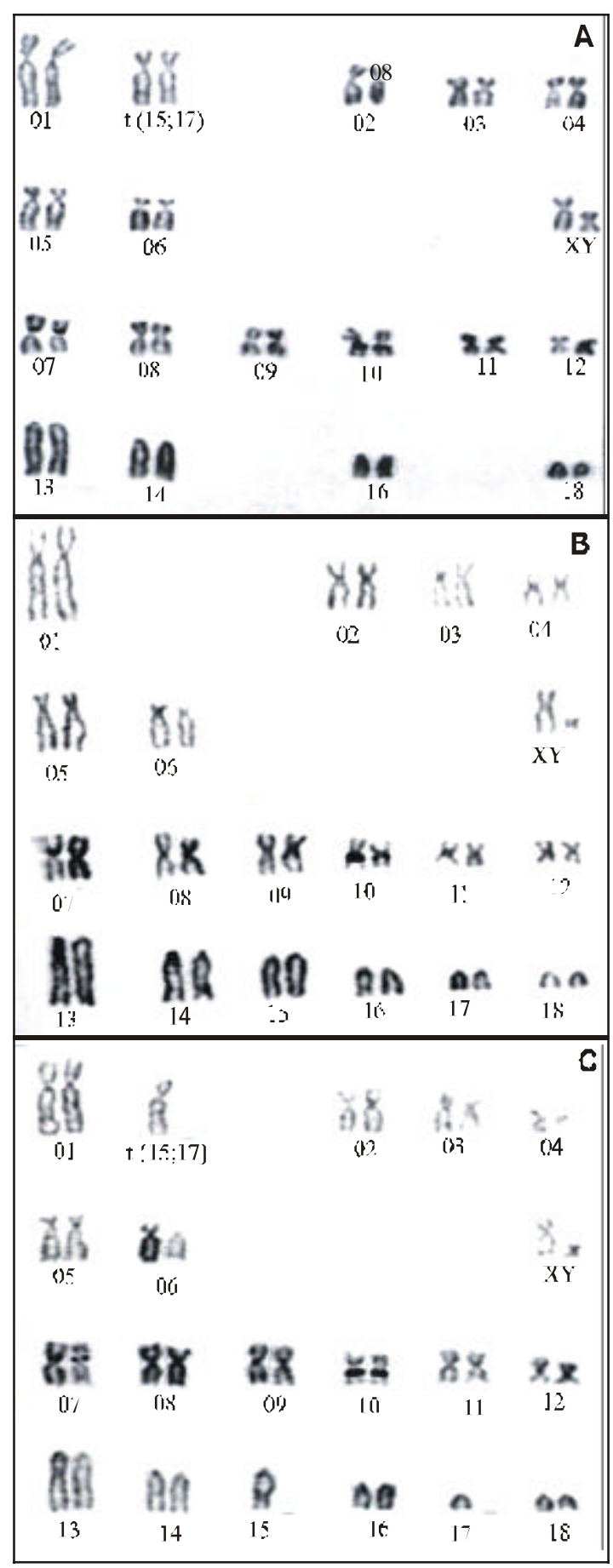

Figura 3. Cariótipo de Sus s. scrofa L. machos, sob bandamento NOR, apresentando $2 \mathrm{n}=36$ cromossomos (A); $2 \mathrm{n}=38$ cromossomos (B) e $2 n=37$ cromossomos $(C)$. 
apenas no par 14, em constrição secundária característica desse par cromossômico, que corresponde ao par 10 nos animais com $2 \mathrm{n}=36,38$ e 37 do presente trabalho.

Foram encontradas variações inter e intraindividuais quanto à intensidade e número de cromossomos marcados pela técnica de Ag-NOR, o que está de acordo com os resultados encontrados por Mellink et al. (1994) com suínos domésticos. Isto pode ser explicado pelo fato de que o método de AgNOR não cora todos os locais de DNAr, mas preferivelmente aqueles locais em que estão ativos transcricionalmente ou que já tenham sido transcritos, pelo menos na intérfase precedente do ciclo celular.

\section{Conclusões}

1. O polimorfismo quanto ao número cromossômico ocorre devido aos cruzamentos entre javalis puros $(2 \mathrm{n}=36)$, suínos domésticos $(2 \mathrm{n}=38)$, javaporcos $(2 n=38)$ e os híbridos $(2 n=37)$.

2. A realização de exames citogenéticos pode ser utilizada na seleção dos acasalamentos.

3. A presença de um cromossomo submetacêntrico médio $\mathrm{t}(15 ; 17)$, formado por fusão robertsoniana, nos animais com $2 n=37$ pode servir para diferenciar javalis híbridos, puros e javaporcos, visto que estes são fenotipicamente muito parecidos.

\section{Agradecimentos}

Aos técnicos João Airton Boer e Paulo Antônio Tosta, do Laboratório de Citogenética Animal, FCAV/ Unesp, pelo auxílio nas coletas de sangue e análises citogenéticas; à Capes e à Unesp, pelo apoio financeiro.

\section{Referências}

ANDERSSON-EKLUND, L.; MARKLUND, L.; LUNDSTROM, K.; HALEY, C. S.; ANDERSSON, K.; HANSSON, I.; MOLLER, M.; ANDERSSON, I. Mapping quantitative trait loci for carcass and meat quality traits in a wild boar x large white intercross. Journal of Animal Science, Albany, v. 76, p. 694-700, 1998.

BOSMA, A. A. Chromosomal polymorphism and $\mathrm{G}$ banding patterns in the wild boar (Sus scrofa L.) from the Netherlands. Genetica, Gravenhage, n. 46, p. 391-399, 1976.
BOSMA, A. A.; HAAN, N. A. de; BLOUCH, R. A.; MacDONALD, A. A. Comparative cytogenetic studies in Sus verrucosus, Sus celebensis and Sus scrofa vittatus (Suidae, Mammalia). Genetica, Gravenhage, v. 83, n. 3, p. 189-194, 1991.

BOSMA, A. A.; HAAN, N. A. de; MELLINK, C. H. M.; YERLE, M.; ZIJLSTRA, C. Chromosome homology between the domestic pig and the babirusa (family Suidae) elucidated with the use of porcine painting probes. Cytogenetics and Cell Genetics, Basel, v. 75, p. 32-35, 1996.

BOSMA, A. A.; OLIVER, W. L. R.; MacDONALD, A. A. The karyotype, including $\mathrm{G}$ and $\mathrm{C}$ banding patterns, of the pigmy hog Sus (Porcula) salvanius (Suidae, Mammalia). Genetica, Gravenhage, v. 61, p. 99-106, 1983.

CHEN, H.; LEIBENGUTH, F. Restriction patterns of mitochondrial DNA in European wild boar and German Landrace. Comparative Biochemistry and Physiology, Oxford, v. 110, n. 4, p. 725-728, 1995.

DARRÉ, R.; BERLANO, H. M.; GOUSTAT, P. Statut chromosomique des populations de sangliers sauvages et d'élevages en France. Revue de Médicine Vétérinaire, Toulouse, v. 143, n. 3, p. 225-232, 1992.

GIANNONI, M. A.; FERRARI, I.; GIANNONI, M. L. Padrões de formação de bandas Ag (bandas pela prata) nos cromossomos das subespécies Sus scrofa scrofa (javali) e Sus scrofa (suíno doméstico). In: SIMPÓSIO NACIONAL TÓPICOS AVANÇADOS EM REPRODUÇÃO ANIMAL, 1., 1980, Jaboticabal. Anais... Jaboticabal: Unesp-FCAV, 1980. p. 27-41.

GIUFFRA, E.; KIJAS, J.; AMARGER, V.; CARLBORG, O.; JEON, J.; ANDERSSON, L. The origin of the domestic pig: independent domestication and subsequent introgression. Genetics, Bethesda, v. 154, p. 1785-1791, 2000.

GOODPASTURE, C.; BLOOM, S. E. Visualization of nucleolar organizer in mammalian chromosomes using silver staining. Chromosoma, Berlin, v. 53, p. 37-50, 1975.

GUSTAVSSON, I. Standard karyotype of the domestic pig. Hereditas, Lund, v. 109, p. 151-157, 1988.

HUANG, Y.; SHI, X.; ZHANG, Y. Mitochondrial genetic variation in Chinese pigs and wild boars. Biochemical Genetics, New York, v. 37, n. 11/12, p. 335-343, 1999. 
LEVAN, A.; FREDGA, K.; SANDBERG, A. A. Nomenclature for centromeric position on chromosomes. Hereditas, Lund, v. 52, p. 201-210, 1964.

McFEE, F. A.; BANNER, M. Inheritance of chromosome number in pigs. Journal of Reproduction and Fertility, Cambridge, Inglaterra, v. 18, p. 9-14, 1969.

McFEE, F. A.; BANNER, M.; RARY, J. Variation in chromosome number among European wild pigs. Cytogenetics, Basel, v. 5, p. 75-81, 1966.

MELLINK, C. H. M.; BOSMA, A. A.; HAAN, N. A. de. Variation in size of Ag-NORs and fluorescent rDNA in situ hybridization signals in six breeds of domestic pig. Hereditas, Lund, v. 120, p. 141-149, 1994.

MOORHEAD, P. S.; HOWELL, P. C.; MELLMAN, W. J.; BATTIPS, D. M.; HUNDGERFORD, D. A.

Chromosome preparations of leukocytes cultured from human peripheral blood. Experimental Cell Research, New York, v. 20, p. 613-616, 1960.

NOWAK, R. M. Walker's mammals of the world. $6^{\text {th }}$ ed. Baltimore: John Hopkins University Press, 1999. v. 2.

PANDA, A. K.; CHATTERJE, A. K.; SAHOO, A. K.; ROY, S.; SAMANTA, S. Chromosome study on a wild boar from Sunderban Tiger Reserve. Indian Journal of Animal Sciences, New Delhi, v. 65, n. 11, p. 1272-1274, 1995.

ROTHSCHILD, M. F.; PLASTOW, G. S. Advances in pig genomics and industry applications. AgBiotechNet, Wallingford, v. 1, p. 1-8, 1999.
SUMNER, A. T. A simple technique for demonstrating centromeric heterochromatin. Experimental Cell Research, New York, v. 75, p. 304-306, 1972.

THOMSEN, P. D.; HOYHEIM, B.; CHRISTENSEN, K. Recent fusion events during evolution of pig chromosomes 3 and 6 identified by comparison with the babirusa karyotype. Cytogenetics and Cell Genetics, Basel, v. 73, n. 3, p. 203-208, 1996.

TIKHONOV, V. N.; TROSHINA, A. Introduction of two chromosomal translocations of Sus scrofa nigripes and Sus scrofa scrofa into the genome of Sus scrofa domestica. Theoretical and Applied Genetics, New York, v. 53, p. 261-264, 1978.

VITIC, J.; STEVANOVIC, J. Comparative studies of the serum lipoprotein and lipids in some domestic, laboratory and wild animals. Comparative Biochemistry and Physiology, Oxford, v. 106, n. 1, p. 223-229, 1993.

WOLKERS, J.; WENSING, T.; BRUINDERINK, G. W. T. A. G.; SCHONEWILLE, A. T. Nutritional status of wild boar (Sus scrofa) - II: body fat reserves in relation to hematology and blood chemistry. Comparative Biochemistry and Physiology, Oxford, v. 105A, n. 3, p. 539-542, 1993.

ZHANG, J.; ROSENBERG, H. F.; NEI, M. Positive Darwinian selection after gene duplication in primate ribonuclease genes. Proceedings of the National Academy of Sciences of the United States of America, Washington, v. 95, p. 3708-3713, 1998. 